\title{
Uniformly convex functions
}

\author{
by Wancang Ma $\ddagger$ and David Minda $\ddagger$ (Cincinnati, OH)
}

\begin{abstract}
Recently, A. W. Goodman introduced the geometrically defined class UCV of uniformly convex functions on the unit disk; he established some theorems and raised a number of interesting open problems for this class. We give a number of new results for this class. Our main theorem is a new characterization for the class UCV which enables us to obtain subordination results for the family. These subordination results immediately yield sharp growth, distortion, rotation and covering theorems plus sharp bounds on the second and third coefficients. We exhibit a function $k$ in UCV which, up to rotation, is the sole extremal function for these problems. However, we show that this function cannot be extremal for the sharp upper bound on the $n$th coefficient for all $n$. We establish this by obtaining the correct order of growth for the sharp upper bound on the $n$th coefficient over the class UCV and then demonstrating that the $n$th coefficient of $k$ has a smaller order of growth.
\end{abstract}

1. Introduction. Goodman $\left[\mathrm{G}_{1}\right]$ introduced the geometrically defined class UCV of uniformly convex functions on the unit disk $\mathbb{D}=\{z:|z|<1\}$. A function $f$ is said to be uniformly convex in $\mathbb{D}$ if $f(z)$ is a normalized $\left(f(0)=f^{\prime}(0)-1=0\right)$ convex function and has the property that for every circular arc $\gamma$ contained in $\mathbb{D}$, with center also in $\mathbb{D}$, the image $\operatorname{arc} f(\gamma)$ is a convex arc. He derived a characterization of uniformly convex functions: a function $f(z)=z+a_{2} z^{2}+a_{3} z^{3}+\ldots$ which is holomorphic on $\mathbb{D}$ belongs to $\mathrm{UCV}$ if and only if for every pair $(z, \zeta)$ in the polydisk $\mathbb{D} \times \mathbb{D}$

$$
1+\operatorname{Re}\left\{(z-\zeta) f^{\prime \prime}(z) / f^{\prime}(z)\right\} \geq 0 .
$$

So far this two-variable characterization has not led to any sharp estimates for the class UCV. The best known bounds on the coefficients for the family $\mathrm{UCV}$ are $\left|a_{n}\right| \leq 1 / n(n=2,3, \ldots)\left[\mathrm{G}_{1}\right]$. Also, Goodman $\left[\mathrm{G}_{1}\right]$ proved that

1991 Mathematics Subject Classification: 30C45.

Key words and phrases: convex functions, growth and distorsion theorems, coefficient bounds.

$\dagger$ Research partially supported by a Taft Graduate Fellowship from the Taft Foundation of the University of Cincinnati.

$\ddagger$ Research partially supported by a National Science Foundation Grant. 
the condition

$$
\sum_{n=2}^{\infty} n(n-1)\left|a_{n}\right| \leq \frac{1}{3}
$$

is sufficient for a function to belong to UCV and that the constant $1 / 3$ is best possible. In $\left[\mathrm{G}_{2}\right]$, Goodman noted that so far there was no reasonable conjecture for the upper bound on $\left|a_{n}\right|$ in the class UCV. One reason for this is that there was no obvious guess for possible extremal functions.

In this paper we introduce a function in the family UCV, which turns out to be extremal for various problems. In order to prove that this function is indeed extremal, we obtain one-variable characterizations for functions in UCV which are closely related to Goodman's characterization. These one-variable characterizations enable us to derive some subordination results for the class UCV which yield sharp distortion, growth, rotation and covering theorems as well as sharp bounds on the second and third coefficients. We also obtain the sharp order of growth for the coefficients. However, the extremal function for the second and third coefficients cannot be extremal for all $n \geq 4$.

After the completion of this paper, we became aware of the related, independent work of Rønning [Rø]. There is some overlap between the two papers; we indicate the duplication at the appropriate places.

2. The class PAR. The class CV of normalized convex univalent functions is closely related to the class $P$ of normalized holomorphic functions with positive real part. Recall that a holomorphic function $p$ defined on $\mathbb{D}$ belongs to $P$ provided that $p(0)=1$ and $\operatorname{Re}\{p(z)\}>0, z \in \mathbb{D}$. Precisely, a normalized locally univalent function $f$ defined on $\mathbb{D}$ is in $\mathrm{CV}$ if and only if $p(z)=1+z f^{\prime \prime}(z) / f^{\prime}(z)$ belongs to $P$. We now introduce a subfamily PAR of $P$ that plays an analogous role for the class UCV. Let

$$
\Omega=\left\{w=u+i v: v^{2}<2 u-1\right\}=\{w: \operatorname{Re} w>|w-1|\} .
$$

Note that $\Omega$ is the interior of a parabola in the right half-plane which is symmetric about the real axis and has vertex at $(1 / 2,0)$. Let

$$
\mathrm{PAR}=\{p \in P: p(\mathbb{D}) \subseteq \Omega\} .
$$

ExAmple 1. It is known $\left[K\right.$, p. 125] that $z=-\tan ^{2}(\sqrt{\omega} \pi / 2 \sqrt{2})$ maps $\left\{\omega=\mu+i v: v^{2}<1-2 \mu\right\}$ conformally onto $\mathbb{D}$. Hence, $z=$ $-\tan ^{2}(\sqrt{1-w} \pi / 2 \sqrt{2})$ maps $\Omega$ conformally onto $\mathbb{D}$. Let $w=q(z)$ be the inverse function. Then $q$ is a Riemann mapping function from $\mathbb{D}$ to $\Omega$ which satisfies $q(0)=1$; more explicitly,

$q(z)=1+\frac{2}{\pi^{2}}\left(\log \frac{1+\sqrt{z}}{1-\sqrt{z}}\right)^{2}=\sum_{n=0}^{\infty} B_{n} z^{n}=1+\frac{8}{\pi^{2}} \sum_{n=1}^{\infty}\left(\frac{1}{n} \sum_{k=1}^{n-1} \frac{1}{2 k+1}\right) z^{n}$. 
Obviously, $q$ belongs to the class PAR. Geometrically, PAR consists of those normalized $(p(0)=1)$ holomorphic functions $p$ defined on $\mathbb{D}$ which are subordinate to $q$, written $p \prec q$. From the elementary inequality

we have the bound

$$
\sum_{k=0}^{n-1} \frac{1}{2 k+1}<1+\frac{1}{2} \log (2 n-1),
$$

for $n \geq 1$.

$$
B_{n}<\frac{8}{n \pi^{2}}\left(1+\frac{1}{2} \log (2 n-1)\right)
$$

There is an analytic characterization of the class PAR that is useful.

THEOREM 1. Suppose $p$ is a normalized $(p(0)=1)$ holomorphic function in $\mathbb{D}$. Then $p \in \mathrm{PAR}$ if and only if $\operatorname{Re}\{p(z)\} \geq|p(z)-1| /|z|$.

Proof. If $p$ satisfies $\operatorname{Re}\{p(z)\} \geq|p(z)-1| /|z|$, then clearly $\operatorname{Re}\{p(z)\} \geq$ $|p(z)-1|$, so $p \in \mathrm{PAR}$.

Next we want to prove that each $p \in \operatorname{PAR}$ satisfies the inequality. It suffices to verify the inequality at any fixed point $a$ in $\mathbb{D}$. Consider any $r$ with $|a|<r<1$. Then for $|z|=r$ we have

$$
\operatorname{Re}\{p(z)\} \geq|p(z)-1|=r|p(z)-1| /|z| \text {. }
$$

Because $\operatorname{Re}\{p(z)\}$ is harmonic and $r|p(z)-1| /|z|$ is subharmonic, we may conclude that the preceding inequality actually holds for $|z|<r$. In particular, we have

$$
\operatorname{Re}\{p(a)\} \geq r|p(a)-1| /|a| .
$$

By letting $r$ tend to 1 we obtain the desired result.

R e mark. The sharp coefficient bounds for functions in the class PAR are immediate consequences of a result of Rogosinski [R] for subordinate functions. Clearly, $q(z)$ is a convex univalent function in $\mathbb{D}$ and $q(\mathbb{D})$ is not a half-plane. His result implies that for $p(z)=1+b_{1} z+b_{2} z^{2}+\ldots \in$ PAR,

$$
\left|b_{n}\right| \leq 8 / \pi^{2}=B_{1} \quad(n=1,2, \ldots)
$$

with equality if and only if $p(z)=q\left(e^{i \theta} z^{n}\right)$ for some $\theta \in \mathbb{R}$.

3. Characterizations of UCV. We present one-variable characterizations of functions in UCV.

THEOREM 2. Assume that $f(z)$ is holomorphic and locally univalent in $\mathbb{D}$ with $f(0)=f^{\prime}(0)-1=0$. Then the following are equivalent:

(i) $f \in \mathrm{UCV}$;

(ii) $1+z f^{\prime \prime}(z) / f^{\prime}(z) \in \operatorname{PAR}$;

(iii) $1+\operatorname{Re}\left\{z f^{\prime \prime}(z) / f^{\prime}(z)\right\} \geq\left|f^{\prime \prime}(z) / f^{\prime}(z)\right|(z \in \mathbb{D})$. 
Proof. Set $p(z)=1+z f^{\prime \prime}(z) / f^{\prime}(z)$. We show that Goodman's characterization of UCV yields (ii). Fix $z \in \mathbb{D}$. For any $r \in(0,1)$ let

$$
\zeta=r \exp \left\{-i \operatorname{Arg} \frac{f^{\prime \prime}(z)}{f^{\prime}(z)}\right\} .
$$

Then from Goodman's characterization of UCV we obtain

$$
1+\operatorname{Re}\left\{z f^{\prime \prime}(z) / f^{\prime}(z)\right\} \geq r\left|f^{\prime \prime}(z) / f^{\prime}(z)\right|,
$$

or $\operatorname{Re}\{p(z)\} \geq r|p(z)-1|$. By letting $r$ tend to 1 , we get the inequality which shows that $p \in \mathrm{PAR}$.

The equivalence of (ii) and (iii) follows from Theorem 1.

Now we verify that (iii) implies (i). From (iii) we have

$$
1+\operatorname{Re}\left\{z f^{\prime \prime}(z) / f^{\prime}(z)\right\} \geq\left|f^{\prime \prime}(z) / f^{\prime}(z)\right| \geq \operatorname{Re}\left\{\zeta f^{\prime \prime}(z) / f^{\prime}(z)\right\}
$$

for any $\zeta \in \mathbb{D}$. Clearly, this is equivalent to Goodman's characterization of UCV.

Note that condition (iii) above appears to be stronger than condition (ii). Also, condition (ii) is sometimes easier to use rather than (iii) in checking whether a function is in UCV. Condition (ii) is analogous to the characterization of convex functions in terms of the class $P$. The equivalence of conditions (i) and (ii) was also established by Rønning [Rø, Thm. 1].

EXAMPLE 2. We now specify a holomorphic function $k$ in $\mathbb{D}$ by $k(0)=$ $k^{\prime}(0)-1=0$ and

$$
1+z k^{\prime \prime}(z) / k^{\prime}(z)=q(z),
$$

where $q$ is the conformal mapping onto $\Omega$ given in Example 1 . Then it is clear from Theorem 2 that $k$ is in UCV. We will see in Sections 4 and 5 that this function is extremal for some problems in the family UCV. Let

$$
k(z)=z+A_{2} z^{2}+A_{3} z^{3}+\ldots
$$

When necessary, we set $A_{1}=1$. From the relationship between the functions $p$ and $k$, we obtain

$$
n(n-1) A_{n}=\sum_{k=1}^{n-1} k A_{k} B_{n-k} .
$$

Since all of the coefficients $B_{n}$ are positive, it follows that all of the coefficients $A_{n}$ are also positive. In particular,

Note that

$$
2 A_{2}=B_{1}=\frac{8}{\pi^{2}}, \quad 6 A_{3}=B_{2}+B_{1}^{2}=\frac{16}{3 \pi^{2}}+\frac{64}{\pi^{4}} .
$$

$$
\log k^{\prime}(z)=\int_{0}^{z} \frac{q(\zeta)-1}{\zeta} d \zeta=\sum_{n=1}^{\infty} \frac{B_{n}}{n} z^{n}
$$


Since

$$
\frac{B_{n}}{n}<\frac{8}{n^{2} \pi^{2}}\left(1+\frac{1}{2} \log (2 n-1)\right),
$$

we see that $\log k^{\prime}$ is continuous in $\overline{\mathbb{D}}$. Therefore, $k$ itself is continuous in $\overline{\mathbb{D}}$.

It is interesting to note that the function $\left(\pi^{2} / 8\right)\left(k^{\prime}(z)-1\right)$ belongs to the class CV. As $q(z)$ is univalent in $\mathbb{D},(q(z)-1) / z \neq 0$ in $\mathbb{D}$. Direct computation gives that

$$
1+\frac{z k^{\prime \prime \prime}(z)}{k^{\prime \prime}(z)}=\frac{z q^{\prime}(z)}{q(z)-1}+q(z)-1 .
$$

Note that $\left(\pi^{2} / 8\right)(q(z)-1)$ is in CV, which implies (see [D, p. 73])

$$
\operatorname{Re}\left\{\frac{z q^{\prime}(z)}{q(z)-1}\right\}>\frac{1}{2} \quad(z \in \mathbb{D}) .
$$

Also note that $\operatorname{Re}\{q(z)-1\}>-1 / 2$ in $\mathbb{D}$. Hence, we have

$$
\operatorname{Re}\left\{1+\frac{z k^{\prime \prime \prime}(z)}{k^{\prime \prime}(z)}\right\}>0 \quad(z \in \mathbb{D}) .
$$

This implies that $\left(\pi^{2} / 8\right)\left(k^{\prime}(z)-1\right)$ is in the class CV.

4. Subordination theorem and consequences. In this section, we first derive some subordination results from Theorem 2; as corollaries we obtain sharp distortion, growth, covering and rotation theorems for the family UCV.

TheOrem 3. Assume that $f \in \mathrm{UCV}$. Then $1+z f^{\prime \prime}(z) / f^{\prime}(z) \prec 1+$ $z k^{\prime \prime}(z) / k^{\prime}(z)$ and $f^{\prime}(z) \prec k^{\prime}(z)$.

Proof. Let $p(z)=1+z f^{\prime \prime}(z) / f^{\prime}(z)$. Then $1+z f^{\prime \prime}(z) / f^{\prime}(z) \prec 1+$ $z k^{\prime \prime}(z) / k^{\prime}(z)$ is the same as $p(z) \prec q(z)$, which follows from Theorem 2(ii). Note that $q(z)-1$ is a convex univalent function in $\mathbb{D}$. By using a result of Goluzin [G] (see also [P, p. 50]) we may conclude that

$$
\log f^{\prime}(z)=\int_{0}^{z} \frac{p(\zeta)-1}{\zeta} d \zeta \prec \int_{0}^{z} \frac{q(\zeta)-1}{\zeta} d \zeta=\log k^{\prime}(z) .
$$

Equivalently, $f^{\prime}(z) \prec k^{\prime}(z)$. This completes the proof of Theorem 3 .

Corollary 1 (Distortion Theorem). Assume $f \in \mathrm{UCV}$ and $|z|=r<1$. Then

$$
k^{\prime}(-r) \leq\left|f^{\prime}(z)\right| \leq k^{\prime}(r) .
$$

Equality holds for some $z \neq 0$ if and only if $f$ is a rotation of $k$.

Pr o of. Since $q(z)-1$ is convex univalent in $\mathbb{D}$, it follows that $\log k^{\prime}(z)$ is also convex univalent in $\mathbb{D}$. In fact, the power series for $\log k^{\prime}(z)$ has positive 
coefficients, so the image of $\mathbb{D}$ under this convex function is symmetric about the real axis. As $\log f^{\prime}(z) \prec \log k^{\prime}(z)$, the subordination principle shows that

$$
\begin{aligned}
k^{\prime}(-r) & =\exp \left\{\log k^{\prime}(-r)\right\}=\exp \left\{\min _{|z|=r} \operatorname{Re} \log k^{\prime}(z)\right\} \\
& \leq \exp \left\{\operatorname{Re} \log f^{\prime}(z)\right\}=\left|f^{\prime}(z)\right| \\
& \leq \exp \left\{\max _{|z|=r} \operatorname{Re} \log k^{\prime}(z)\right\}=\exp \left\{\log k^{\prime}(r)\right\}=k^{\prime}(r) .
\end{aligned}
$$

Note that for $\left|z_{0}\right|=r$, either

$$
\operatorname{Re}\left\{\log f^{\prime}\left(z_{0}\right)\right\}=\min _{|z|=r} \operatorname{Re}\left\{\log k^{\prime}(z)\right\}
$$

or

$$
\operatorname{Re}\left\{\log f^{\prime}\left(z_{0}\right)\right\}=\max _{|z|=r} \operatorname{Re}\left\{\log k^{\prime}(z)\right\}
$$

for some $z_{0} \neq 0$ if and only if $\log f^{\prime}(z)$ is $\log k^{\prime}\left(e^{i \theta} z\right)$ for some $\theta \in \mathbb{R}$; this follows from the principle of subordination. Therefore, the equality assertion holds. This completes our proof. where

Rønning [Rø, Thm. 6] obtains the uniform upper bound $\left|f^{\prime}(z)\right| \leq N$,

$$
N=\exp \left(\frac{14}{\pi^{2}} \zeta(3)\right)=5.502 \ldots,
$$

and $\zeta(t)$ denotes the Riemann Zeta function.

By making use of standard techniques and Corollary 1, we obtain the following growth and covering theorems.

Corollary 2 (Growth Theorem). Let $f \in \mathrm{UCV}$ and $|z|=r<1$. Then

$$
-k(-r) \leq|f(z)| \leq k(r) .
$$

Equality holds for some $z \neq 0$ if and only if $f$ is a rotation of $k$.

Corollary 3 (Covering Theorem). Suppose $f \in \mathrm{UCV}$. Then either $f$ is a rotation of $k$ or $f(\mathbb{D}) \supseteq\{w:|w| \leq-k(-1)\}$.

Recall that $k$ is continuous on $\overline{\mathbb{D}}$ so $k(-1)$ makes sense. Also, $-k(-1)<$ $k(1)$ since the power series for $k$ has positive coefficients. From Corollary 2 it follows that the functions in UCV are uniformly bounded above by the sharp constant $k(1)$. The following rotation theorem follows from the subordination $f^{\prime} \prec k^{\prime}$ given in Theorem 3 .

COROllary 4 (Rotation Theorem). Let $f \in \mathrm{UCV}$ and $\left|z_{0}\right|=r<1$. Then

$$
\left|\operatorname{Arg}\left\{f^{\prime}\left(z_{0}\right)\right\}\right| \leq \max _{|z|=r} \operatorname{Arg}\left\{k^{\prime}(z)\right\} .
$$

Equality holds for some $z_{0} \neq 0$ if and only if $f$ is a rotation of $k$. 
5. Coefficient bounds. We first give sharp bounds on the second and third coefficients for functions in UCV. Then we obtain the sharp order of growth for the coefficients of functions in UCV.

For $f(z)=z+a_{2} z^{2}+a_{3} z^{3}+\ldots \in \mathrm{UCV}$, define

$$
p(z)=1+z f^{\prime \prime}(z) / f^{\prime}(z)=1+b_{1} z+b_{2} z^{2}+\ldots
$$

Then

$$
n(n-1) a_{n}=\sum_{k=1}^{n-1} k a_{k} b_{n-k} .
$$

In particular, $2 a_{2}=b_{1}$ and $6 a_{3}=b_{2}+b_{1}^{2}$. Recall the analogous expressions related to $k$ and $q$ that were given in Examples 1 and 2 .

THEOREM 4. Let $f(z)=z+a_{2} z^{2}+a_{3} z^{3}+\ldots \in \mathrm{UCV}$. Then we have the sharp bounds

$$
\left|a_{2}\right| \leq A_{2}=4 / \pi^{2}, \quad\left|a_{3}\right| \leq A_{3}=8 /\left(9 \pi^{2}\right)+32 /\left(3 \pi^{4}\right) .
$$

Equality holds in either inequality if and only if $f$ is a rotation of $k$.

Proof. Since $p \prec q$, we have $\left|b_{1}\right| \leq B_{1}$ with equality if and only if $p(z)$ is $q\left(e^{i \theta} z\right)$ for some $\theta \in \mathbb{R}$. This implies that $\left|a_{2}\right| \leq A_{2}$ with equality if and only if $f$ is a rotation of $k$. Rønning [Rø, Thm. 5] also obtained this bound.

Moreover, a result of Rogosinski for subordinate functions $([\mathrm{R}]$, see also [D, p. 192]) gives

So

$$
\left|b_{1}\right|^{2}+\left|b_{2}\right|^{2} \leq B_{1}^{2}+B_{2}^{2}
$$

$$
\begin{aligned}
6\left|a_{3}\right| & \leq\left|b_{1}\right|^{2}+\left|b_{2}\right| \leq\left|b_{1}\right|^{2}+\frac{1}{2} B_{2}\left(1+\left|b_{2}\right|^{2} / B_{2}^{2}\right) \\
& =\frac{1}{2} B_{2}+\left(1-1 /\left(2 B_{2}\right)\right)\left|b_{1}\right|^{2}+\left(\left|b_{1}\right|^{2}+\left|b_{2}\right|^{2}\right) /\left(2 B_{2}\right) \\
& \leq \frac{1}{2} B_{2}+\left(1-1 /\left(2 B_{2}\right)\right) B_{1}^{2}+\left(B_{1}^{2}+B_{2}^{2}\right) /\left(2 B_{2}\right)=B_{2}+B_{1}^{2}=6 A_{3} .
\end{aligned}
$$

Note that we have employed the inequality $\left|b_{1}\right| \leq B_{1}$ here, so equality holds if and only if $f$ is a rotation of $k$. This completes the proof of Theorem 4 .

In order to give the sharp order of growth for the coefficients of a function in UCV, we shall need the following example.

EXAMPLE 3. We define holomorphic functions $k_{n}(z)(n=2,3, \ldots)$ in the unit disk $\mathbb{D}$ by $k_{n}(0)=k_{n}^{\prime}(0)-1=0$ and

$$
1+\frac{z k_{n}^{\prime \prime}(z)}{k_{n}^{\prime}(z)}=q\left(z^{n-1}\right),
$$

where $q(z)$ is given in Example 1. It is clear that $k_{2}(z)=k(z)$ and

$$
k_{n}(z)=z+\frac{8}{n(n-1) \pi^{2}} z^{n}+\ldots
$$


This implies the lower bound

$$
\max _{f \in \mathrm{UCV}}\left|a_{n}\right| \geq \frac{8}{n(n-1) \pi^{2}} .
$$

Rønning [Rø, Cor. 1 of Thm. 2] showed that the function $f(z)=z+\alpha z^{n} \in$ $\mathrm{UCV}$ if and only if $|\alpha| \leq 1 /(n(2 n-1))$; this result is due to Goodman $\left[\mathrm{G}_{1}\right]$ in the case $n=2$. Note that $8 /\left(n(n-1) \pi^{2}\right)>1 /(n(2 n-1))$ for every $n=2,3, \ldots$

TheOREM 5. Let $f(z)=z+a_{2} z^{2}+a_{3} z^{3}+\ldots \in \mathrm{UCV}$. Then we have the sharp order of growth $\left|a_{n}\right|=O\left(1 / n^{2}\right)$.

Proof. From Example 3, we see that this order is best possible. We now show that there exists a constant $M$ such that $n^{2}(n-1)^{2}\left|a_{n}\right|^{2} \leq M^{2}$. Because $p \prec q$ and $f^{\prime} \prec k^{\prime}$, we have

$$
\sum_{k=1}^{n-1}\left|b_{k}\right|^{2} \leq \sum_{k=1}^{n-1} B_{k}^{2}
$$

and

$$
1+\sum_{k=2}^{n-1} k^{2}\left|a_{k}\right|^{2} \leq 1+\sum_{k=2}^{n-1} k^{2} A_{k}^{2}
$$

by Rogosinski's result ([R], see also [D, p. 192]). From

$$
n(n-1) a_{n}=\sum_{k=1}^{n-1} k a_{k} b_{n-k}
$$

we obtain

$$
\begin{aligned}
n^{2}(n-1)^{2}\left|a_{n}\right|^{2} & \leq\left(\sum_{k=1}^{n-1}\left|b_{k}\right|^{2}\right)\left(1+\sum_{k=2}^{n-1} k^{2}\left|a_{k}\right|^{2} \mid\right) \\
& \leq\left(\sum_{k=1}^{n-1} B_{k}^{2}\right)\left(1+\sum_{k=2}^{n-1} k^{2} A_{k}^{2}\right)
\end{aligned}
$$

by using the Cauchy-Schwarz inequality and the Rogosinski-type results. From the bound on $B_{k}$ given in Example 1, we have

$$
\sum_{k=1}^{\infty} B_{k}^{2}<\frac{64}{\pi^{4}} \sum_{k=1}^{\infty} k^{-2}\left(1+\frac{1}{2} \log (2 k-1)\right)^{2}<\infty .
$$

Furthermore, observe that

$$
1+\sum_{k=2}^{\infty} k A_{k} z^{k-1}=k^{\prime}(z)=\exp \left\{\sum_{k=1}^{\infty} k^{-1} B_{k} z^{k}\right\} .
$$


Then an inequality of Milin (see $[\mathrm{P}$, p. 81]) gives

$$
1+\sum_{k=2}^{\infty} k^{2} A_{k}^{2} \leq \exp \left\{\sum_{k=1}^{\infty} k^{-1} B_{k}^{2}\right\}<\infty .
$$

The proof of Theorem 5 is complete if we set

$$
M^{2}=\left(\sum_{k=1}^{\infty} B_{k}^{2}\right)\left(1+\sum_{k=2}^{\infty} k^{2} A_{k}^{2}\right) .
$$

Rønning's work [Rø, Thm. 5] implies our Theorem 5. Since we also employ our method of proof in Section 6, we have included our proof.

R e m a rk. We know that $f^{\prime}(z)-1 \prec k^{\prime}(z)-1$ from Theorem 3 and that $\left(\pi^{2} / 8\right)\left(k^{\prime}(z)-1\right)$ is in the class CV from Example 2. Rogosinski's result [R] for subordinate functions implies that if $f(z)=z+a_{2} z^{2}+a_{3} z^{3}+\ldots \in \mathrm{UCV}$, then $n\left|a_{n}\right| \leq 2 A_{2}=8 / \pi^{2}(n=2,3, \ldots)$. Moreover,

$$
\begin{aligned}
n(n-1)\left|a_{n}\right| & \leq \sum_{k=1}^{n-1} k\left|a_{k}\right|\left|b_{n-k}\right| \\
& =\left|b_{n-1}\right|+\sum_{k=2}^{n-1} k\left|a_{k}\right|\left|b_{n-k}\right| \leq \frac{8}{\pi^{2}}+\frac{64}{\pi^{4}}(n-2) .
\end{aligned}
$$

Here we have used the bound $\left|b_{k}\right| \leq 8 / \pi^{2}$ which is given in Section 2 . Therefore, we have for $n \geq 4$,

$$
n\left|a_{n}\right| \leq \frac{8}{3 \pi^{2}}+\frac{128}{3 \pi^{4}}<0.71
$$

which improves Goodman's result that $n\left|a_{n}\right| \leq 1\left[\mathrm{G}_{1}\right]$. This upper bound can be slightly improved by iterating the preceding method.

6. Open problems. It might seem that Theorem 4 suggests that the coefficients of the function $k$ provide sharp upper bounds on all coefficients for the class UCV. Unfortunately, this nice situation cannot be the case for all $n$ sufficiently large since we can show that $A_{n}=O\left((\log n)^{2} / n^{3}\right)$ as $n \rightarrow \infty$. From the proof of Theorem 5 , we know that there is a finite constant $M$ such that $n(n-1) A_{n} \leq M$ for all $n$. Hence we have

$$
\begin{aligned}
n(n-1) A_{n}= & \sum_{k=1}^{n-1}(n-k) A_{n-k} B_{k} \leq B_{n-1}+M \sum_{k=1}^{n-2} \frac{1}{n-k-1} B_{k} \\
< & \frac{8}{(n-1) \pi^{2}}\left(1+\frac{1}{2} \log (2 n-3)\right) \\
& +\frac{8 M}{\pi^{2}} \sum_{k=1}^{n-2} \frac{1}{k(n-k-1)}\left(1+\frac{1}{2} \log (2 k-1)\right)
\end{aligned}
$$




$$
\begin{aligned}
= & \frac{8}{(n-1) \pi^{2}}\left\{1+\frac{1}{2} \log (2 n-3)\right. \\
& \left.+M \sum_{k=1}^{n-2} \frac{1}{k}\left(2+\frac{1}{2} \log (2 k-1)+\frac{1}{2} \log (2 n-2 k-3)\right)\right\} \\
< & \frac{8}{(n-1) \pi^{2}}\left\{1+\frac{1}{2} \log (2 n-3)+M(2+\log (2 n-5)) \sum_{k=1}^{n-2} \frac{1}{k}\right\} \\
< & \frac{8}{(n-1) \pi^{2}}\left(1+\frac{1}{2} \log (2 n-3)\right)(1+2 M+M \log (2 n-5)),
\end{aligned}
$$

which tends to zero as $n$ tends to infinity.

Therefore, for the class UCV plausible sharp coefficient bounds and associated extremal functions for all $n \geq 4$ remain open problems. Also, what is the smallest value of $n$ such that the $n$th coefficient of $k$ does not provide the sharp upper bound on the $n$th coefficient over the class UCV? Perhaps as $n$ increases, the extremal function for the $n$th coefficient might change from $k(z)=k_{2}(z)$ to $k_{m}(z)$ with $2<m \leq n$.

It would be of interest to give an internal geometric characterization of uniformly convex regions. Specifically, a region $\Omega$ in the complex plane is called uniformly convex if $\Omega=f(\mathbb{D})$ for some function $f$ in UCV. Can one find an internal geometric condition on a convex region $\Omega$ containing the origin which is necessary and sufficient for the Riemann mapping function $f$ of $\mathbb{D}$ onto $\Omega$ which satisfies $f(0)=0$ to belong to UCV after possible normalization of the derivative at the origin? Since the class UCV is not linearly invariant $\left[\mathrm{G}_{1}\right]$ it seems that the base point 0 in $\Omega$ must play some role in this geometric characterization. Also, the characterization of uniformly convex regions should be invariant under rotations about the origin and stretchings.

Acknowledgements. We wish to thank A. W. Goodman for bringing the work of F. Rønning to our attention and R. Barnard for a useful suggestion.

\section{References}

[D] P. Duren, Univalent Functions, Grundlehren Math. Wiss. 259, Springer, New York 1983.

[G] G. M. Goluzin, On the majorization principle in function theory, Dokl. Akad. Nauk SSSR 42 (1935), 647-650 (in Russian).

$\left[\mathrm{G}_{1}\right]$ A. W. Goodman, On uniformly convex functions, Ann. Polon. Math. 56 (1991), $87-92$.

$\left[\mathrm{G}_{2}\right]-$, Coefficient problems in geometric function theory, to appear. 
[K] H. Kober, Dictionary of Conformal Representations, Dover, New York 1957.

[P] Ch. Pommerenke, Univalent Functions, Vandenhoeck \& Ruprecht, Göttingen 1975.

[R] W. Rogosinski, On the coefficients of subordinate functions, Proc. London Math. Soc. 48 (1943), 48-82.

[Rø] F. Rønning, Uniformly convex functions and a corresponding class of starlike functions, Proc. Amer. Math. Soc., to appear.

DEPARTMENT OF MATHEMATICAL SCIENCES

UNIVERSITY OF CINCINNATI

CINCINNATI, OHIO 45221-0025

U.S.A.

Reçu par la Rédaction le 6.9.1991

Added in proof (May 1992). In the notation of Theorem 4, we can now show that $\left|a_{4}\right| \leq A_{4}$. The open problems in Section 6 should be modified accordingly. 